\title{
Morbidity and mortality profile of neonates admitted in a special care newborn unit of a tertiary care teaching hospital of Assam, India
}

\author{
Kutubur Rahman, Rukeya Begum \\ Corresponding author: Dr Kutubur Rahman, Assistant Professor, Department of Pediatrics, \\ Tezpur Medical College, Tezpur, Assam, India; Email - drkutuburrahman@gmail.com \\ Distributed under Attribution-Non Commercial - Share Alike 4.0 International (CC BY-NC-SA 4.0)
}

\begin{abstract}
$\underline{\text { ABSTRACT }}$
Objectives: The objective of study is to estimate the morbidity and mortality of newborns admitted at Special Care Newborn Unit (SCNU) of tertiary care hospital. Materials and methods: This hospital based retrospective study was carried out in SCNU, department of pediatrics, over a period of 4 years from ${ }^{\text {st }}$ January 2016 to $31^{\text {st }}$ December 2019. All newborns (0- 28 days) admitted into the SCNU during the study period were included in the study. Data were collected from the monthly reporting format and patient case records. Then they were compiled in MS excel and analyzed using appropriate statistical tools. Results: During this period of 4 years total 5649 number of patients were admitted in SCNU, inborn 3657 (64.7\%) and out born 1992 (35.2\%). 58.7\% of babies were male. Almost equal number of normal weight $(50.2 \%)$ and low birth weight babies $(49.7 \%)$ were admitted. Similarly equal number of term (49.4\%) and preterm babies (50.5\%) were admitted. Birth asphyxia, babies with birth weight below $1800 \mathrm{gm}$ requiring special care, neonatal sepsis and jaundice requiring phototherapy were common morbidities requiring admission. Mortality was $11.4 \%$ which is higher in out born $(14.3 \%)$ than inborn $(9.9 \%)$. Birth asphyxia $(53.9 \%)$ is the commonest cause of mortality followed by respiratory distress syndrome (RDS) with prematurity $(23.2 \%)$ and neonatal sepsis (12.4\%). Sepsis is higher in out born unit $(18.5 \%)$ than inborn $(7.7 \%)$. Highest number of death occurs in early neonatal period $(88.5 \%)$ and among low birth weight baby $(58.7 \%)$. First 72 hours of admission is crucial for survival as $78.2 \%$ babies die during this period. Conclusion: Birth asphyxia, RDS with prematurity and neonatal sepsis are common cause of mortality. It is largely preventable by comprehensive antenatal, natal, neonatal care and health awareness among patients and society.
\end{abstract}

Keywords: Morbidity, mortality, neonates, inborn, out born, SCNU.

Globally around 15000 babies die every day before attaining their $5^{\text {th }}$ birth day ${ }^{1}$. Out of this around half of the babies are below one month of age. In terms of absolute numbers this translates to 7000 death every day and 26 lakhs death every year ${ }^{1}$. In India alone 6.4 lakh babies die every year below one moth of age which is $24 \%$ of global burden ${ }^{1}$.
National Family Health Survey-4 (NFHS-4) ${ }^{2}$ which was conducted during 2015-16 was published on January 2018. The data reveals under five mortality as 49.5 , infant mortality rate as 40.7 and neonatal mortality rate as 29.5 per thousand live birth with wide range of variation among the states. Kerala has achieved best healthcare indices (U5MR 7.1, IMR 5.6, NMR 4.4) while highest mortality figures are

Received: $25^{\text {th }}$ February 2020. Accepted: $28^{\text {th }}$ April 2020.

Rahman K, Begum R. Morbidity and mortality profile of neonates admitted in a special care newborn unit of a tertiary care teaching hospital of Assam, India. The New Indian Journal of OBGYN. 2020; 7(1): 82-7. 
from Uttar Pradesh (U5MR 78.1, IMR 63.5, NMR 45.1). Our state Assam is still above the national average which is under 5 mortality 56.5 , infant mortality 47.6 and neonatal mortality 32.8 per thousand live births. Above study also reveals that $60 \%$ of under five deaths occur in the neonatal period.

Therefore a lot of initiative has been taken by Government of India to reduce the neonatal mortality. Facility based newborn care is established in 3 levels. Level I care is at first referral unit as neonatal stabilization unit (NBSU), level II care as special care newborn unit (SCNU) at district hospital and level III care at regional level. New national health policy ${ }^{4}$ was announced on 2017 with the goal of reducing under-five mortality rate (U5MR) to 23 , infant mortality rate (IMR) to 28, and neonatal mortality rate (NMR) to 16 by 2025. Our study region caters patients from nearby 6 districts of upper and middle part of Assam on the north bank, one district on the south bank and part of Arunachal Pradesh. Review of the literature reveals that there is no data regarding profiles of newborn from this area. Therefore a retrospective study was carried out to know the mortality and morbidity profiles of the newborns so that if required health policy can be modified in future which will be specific to the area.

\section{Materials and methods}

This retrospective hospital based study was carried out in SCNU, department of pediatrics, in tertiary

Table 2: Admission profile bases on gender, birth weight and gestation of neonates

\begin{tabular}{lllll}
\hline Categories & & $\begin{array}{l}\text { In born } \\
\text { N=3657 }\end{array}$ & $\begin{array}{l}\text { Out born } \\
\mathbf{N = 1 9 9 2}\end{array}$ & $\begin{array}{l}\text { Total } \\
\text { N=5649 }\end{array}$ \\
\hline \multirow{2}{*}{ Gender } & Male & $2136(58.4 \%)$ & $1183(59.3 \%)$ & $3319(58.7 \%)$ \\
& Female & $1521(41.5 \%)$ & $811(40.7 \%)$ & $2332(41.2 \%)$ \\
\hline \multirow{3}{*}{ Birth } & $>2500 \mathrm{gm}$ & $1861(50.8 \%)$ & $976(46.5 \%)$ & $2837(50.2 \%)$ \\
weight & $1500-2499 \mathrm{gm}$ & $1507(41.2 \%)$ & $721(36.1 \%)$ & $2228(39.4 \%)$ \\
& $1000-1499 \mathrm{gm}$ & $259(7.0 \%)$ & $260(13.05 \%)$ & $519(9.1 \%)$ \\
& $<1000 \mathrm{gm}$ & $30(0.8 \%)$ & $35(1.7 \%)$ & $65(1.15 \%)$ \\
\hline \multirow{3}{*}{ Gestation } & $>37 \mathrm{wks}$ & $1819(49.7 \%)$ & $973(48.8 \%)$ & $2792(49.4 \%)$ \\
& $34-37 \mathrm{wks}$ & $1256(34.3 \%)$ & $618(31.02 \%)$ & $1874(33.1 \%)$ \\
& $<34 \mathrm{wks}$ & $582(15.9 \%)$ & $401(20.1 \%)$ & $983(17.4 \%)$ \\
\hline
\end{tabular}
care hospital from $1^{\text {st }}$ January 2016 to $31^{\text {st }}$ December 2019. All newborns (0- 28 days) admitted into the SCNU during the study period were included in the study. The SCNU has total 20 beds with 15 inborn and 5 out born beds. It is equipped with 20 radiant warmers, 12 phototherapy units, 4 (continuous positive airway pressure) CPAP and one ventilator. Hospital stay, drugs and investigations are provided free of cost to all babies in SCNU. Data were collected from the monthly record and patient files and compiled in MS excel. Data were analyzed using appropriate statistical tools.

\section{Results}

During study period, 30377 deliveries were performed in the hospital with an average 7595 per year. Caesarean section comprised of $54.5 \%$. Total delivery was increased by $11.2 \%$ while caesarean section was increased by $14.6 \%$ at

delivery load in the hospital

$\begin{array}{llllll}\mathbf{2 0 1 6} & \mathbf{2 0 1 7} & \mathbf{2 0 1 8} & \mathbf{2 0 1 9} & \text { Total } & \text { Parameters } \\ 7113 & 7396 & 7854 & 8014 & 30377 & 7595 / \mathrm{yr} \\ 3730 & 4135 & 4345 & 4372 & 16582 & 54.5 \% \\ 6777 & 7086 & 7592 & 7681 & 29136 & 7284 / \text { year } \\ 336 & 310 & 262 & 348 & 1256 & 4.1 \% \\ 1881 & 1564 & 1622 & 1681 & 6748 & 22.2 \%\end{array}$

ment caesarian section, LBW = Low birth weight

2019 in comparison to 2016 with increase in total deliveries and caesarean section every year. Live birth was 29136 with average 7284/year and still birth was 1256 which was $4.1 \%$ of total deliveries. Incidence of low birth weight (birth weight $<2500 \mathrm{gm})$ newborn is $6748(22.2 \%)$ of total deliveries (table 1). During this period of 4 years total 5649 number of patients were admitted in SCNU, inborn 3657 (64.7\%) and out born 1992 (35.2\%). Male babies were admitted more, overall male $58.7 \%$ and female $41.2 \%$. Almost equal number of male babies was admitted in out born and inborn unit (59.3\% vs $58.4 \%)$. Similarly equal number $(50.2 \%$ vs $49.7 \%)$ of normal weight and low birth weight babies were admitted in the SCNU though in out born unit normal weight babies (46.5\%) were less than low birth weight. Among LBW babies, babies between 1500-2499gm were $39.4 \%$, 1000-1499gm (Very low birth weight) was 9.1\% and below 1000gm (Extremely low birth weight) was $1.15 \%$. In out born unit more very low birth weight (VLBW) $(13.05 \%$ vs $7 \%)$ and extremely low birth weight (ELBW) $(1.7 \%$ vs $0.8 \%)$ babies were admitted. As per gestation almost equal number of term $(49.4 \%)$ preterm $(50.57 \%)$ were admitted. In out born unit babies $<34$ wks were admitted more (20.1\%vs15.9\%). These findings were shown in the table 2. Both in the inborn and out born unit birth asphyxia was the commonest cause of admission. In inborn unit it was $27.3 \%$ while in out born unit $31.2 \%$. It was 
Table 3: Morbidity profile

\begin{tabular}{llll}
\hline Disease & Inborn & Outborn & Total \\
\hline Preterm RDS & $176(4.8 \%)$ & $121(6.0 \%)$ & $297(5.25 \%)$ \\
MAS & $229(6.2 \%)$ & $33(1.6 \%)$ & $262(4.6 \%)$ \\
Other causes of Respiratory distress & $77(2.1 \%)$ & $51(2.56 \%)$ & $128(2.26 \%)$ \\
Birth Asphyxia & $999(27.3 \%)$ & $623(31.2 \%)$ & $1622(28.7 \%)$ \\
Sepsis & $354(9.6 \%)$ & $258(12.9 \%)$ & $612(10.8 \%)$ \\
Congenital malformation & $22(0.06 \%)$ & $17(0.08 \%)$ & $39(0.69 \%)$ \\
Jaundice & $753(20.5 \%)$ & $372(18.6 \%)$ & $1125(19.9 \%)$ \\
Hypothermia & $54(1.4 \%)$ & $26(1.3 \%)$ & $80(1.4 \%)$ \\
Hypoglycemia & $176(4.8 \%$ & $34(1.7 \%)$ & $2103.7 \%)$ \\
Other & $817(22.3 \%)$ & $457(22.9 \%)$ & $1269(22.4 \%)$ \\
\hline Total & 3657 & 1992 & 5649 \\
\hline RDS - Respiratory distress syndrome & MAS - Meconium aspiration syndrome
\end{tabular}

RDS - Respiratory distress syndrome, MAS - Meconium aspiration syndrome aspiration syndrome (MAS) and transient tachypnea of the newborn (TTNB) were $6.2 \%$ and $2.1 \%$ respectively. Overall RDS among preterm babies is 176 out of 1838 (9.5\%). Among out born respiratory morbidities, it was preterm RDS $6 \%$, MAS $1.6 \%$, other like TTNB 2.5\%. Overall preterm RDS is 121 out of $1019(11.8 \%) \quad($ table 3). Congenital malformation is admitted only $0.06 \%$ and $0.08 \%$ in inborn and out born respectively. It is because the hospital did not have facility of pediatric surgery, so the babies with congenital malformation were to be referred. In inborn unit $80.7 \%$ of patients were discharged successfully while $7.2 \%$ patients left the SCNU against medical advice and we referred $2 \%$ of patients for better treatment which is not available at the hospital. Similarly in out born unit $71.5 \%$ of patients were discharged while $9.8 \%$ patients left against medical advice and jaundice requiring phototherapy which was $20.5 \%$ and $18.6 \%$ in inborn and out born respectively. Neonatal sepsis was $9.6 \%$ in inborn unit but higher in out born unit $12.9 \%$. Among the respiratory morbidities, in inborn unit preterm respiratory distress syndrome (RDS) was $4.8 \%$. Meconium

Table 5: Mortality profile based on gender, birth weight, gestation age at death and duration between admission and death

\begin{tabular}{lllll}
\hline Categories & & $\begin{array}{l}\text { In born } \\
\mathbf{N}=\mathbf{3 6 3}\end{array}$ & $\begin{array}{l}\text { Out born } \\
\mathbf{N = 2 8 6}\end{array}$ & $\begin{array}{l}\text { Total } \\
\mathbf{N}=\mathbf{6 4 9}\end{array}$ \\
\hline Gender & Male & $215(59.2 \%)$ & $166(58.0 \%)$ & $381(58.7 \%)$ \\
& Female & $148(40.7 \%)$ & $120(41.9 \%)$ & $268(41.2 \%)$ \\
\hline \multirow{3}{*}{ Birth weight } & $>2500 \mathrm{gm}$ & $149(40.04 \%)$ & $119(41,6 \%)$ & $268(41.2 \%)$ \\
& $1500-2499 \mathrm{gm}$ & $139(38.2 \%)$ & $90(31.4 \%)$ & $229(35.2 \%)$ \\
& $1000-1499 \mathrm{gm}$ & $58(15.9 \%)$ & $57(19.9 \%)$ & $115(17.7 \%)$ \\
& $<1000 \mathrm{gm}$ & $17(4.6 \%)$ & $20(6.9 \%)$ & $37(5.7 \%)$ \\
\hline Gestation & Term & $206(56.5 \%)$ & $162(56.6 \%)$ & $368(56.7 \%)$ \\
& Preterm & $157(43.2 \%)$ & $124(43.3 \%)$ & $281(43.2 \%$ \\
\hline \multirow{3}{*}{ Age at death } & $<1$ day & $71(19.5 \%)$ & $20(6.9 \%)$ & $91(14.0 \%)$ \\
& $1-7$ days & $265(73.0 \%)$ & $219(76.5 \%)$ & $484(74.5 \%)$ \\
\hline Duration between & $>7$ days & $27(7.4 \%)$ & $47(16.4 \%)$ & $74(11.4 \%)$ \\
admission and & $<1$ day & $97(26.7 \%)$ & $81(28.3 \%)$ & $178(27.4 \%)$ \\
death & $1-3$ days & $189(52.0 \%)$ & $141((49.3 \%)$ & $330(50.8 \%)$ \\
& $4-7$ days & $58(15.9 \%)$ & $43(15.0 \%)$ & $101(15.5 \%)$ \\
& $>7$ days & $19(5.2 \%)$ & $21(7.3 \%)$ & $40(6.1 \%)$ \\
\hline
\end{tabular}
we had to refer $4.2 \%$ of patients to higher facility for treatment which is not available at the hospital mainly for surgical interventions (table 4). Mortality is overall 649 out of $5649(11.4 \%)$ where as in inborn unit it is 363 out of $3657(9.9 \%)$ and out born 286 out of 1992 $(14.3 \%)$. Among the 649 deaths $381(58.7 \%)$ were male and 268 (41.2\% were female). In inborn unit 59.2\% Male and $40.7 \%$ female baby were admitted where as in out born unit male was $58.0 \%$ and female was $41.9 \%$. In both units male mortality is higher than female. Term babies were more than Preterm (56.7\% vs. $43.2 \%) .14 \%$ of babies 
died within 24 hours of birth while $74.5 \%$ died between 1-7 days resulting in cumulative early neonatal death $88.5 \%$ and late neonatal death (after 7 days of age) was $11.4 \%$. Among the death, $58.7 \%$ were low birth weight babies and only $41.2 \%$ were of normal weight. Among low birth weight, $35.2 \%$ was in between $1500-2499 \mathrm{gm}, 17.7 \%$ was between 1000-1499gm and 5.7\% was below 1000gm. Death after

Table 6: Disease specific mortality

\begin{tabular}{llll}
\hline Disease & $\begin{array}{l}\text { Inborn } \\
\mathbf{n = 3 6 3}\end{array}$ & $\begin{array}{l}\text { Outborn } \\
\mathbf{n = 2 8 6}\end{array}$ & $\begin{array}{l}\text { Total } \\
\mathbf{n = 6 4 9}\end{array}$ \\
\hline Prematurity with RDS & $81(22.3 \%)$ & $70(24.4 \%)$ & $151(23.2 \%)$ \\
MAS & $17(4.6 \%)$ & $4(1.39 \%)$ & $21(3.2 \%)$ \\
Birth asphyxia & $210(57.8 \%)$ & $140(48.9 \%)$ & $350(53.9 \%)$ \\
Sepsis & $28(7.7 \%)$ & $53(18.5 \%)$ & $81(12.4 \%)$ \\
Congenital malformation & $1(0.27 \%)$ & 0 & $1(0.15 \%)$ \\
Others & $25(6.8 \%)$ & $19(6.6 \%)$ & $44(6.7 \%)$ \\
Cause not established & $1 \quad(0.27 \%)$ & 0 & $1(0.15 \%)$ \\
\hline
\end{tabular}

taking admission to the SCNU took place in $27.4 \%$ of cases within 24 hours where as between 1-3 days 50.8\%, 4-7 days $15.5 \%$. Beyond 7 days of admission it was only in $6.1 \%$. Thus among death babies, $78.2 \%$ of the babies died within

though in other study there were more number of low birth weight neonates like Anuradha D et al $74.2 \%$ and Saharia $\mathrm{NP}$ et al $60 \%$. In our study term neonate admission is $49.4 \%$

$\mathrm{N} \mathrm{P}$ et $\mathrm{al}^{5}$ ( inborn 59.3\%. out born 40.7\%), and Malik S et $\mathrm{al}^{8}$ (inborn $57.2 \%$,and outborn $42.8 \%$ ). $58.7 \%$ of the neonates were male and $41.2 \%$ were female which is similar to the studies done by Baruah $\mathrm{M} \mathrm{N}$ et $\mathrm{al}^{7}$ (58.4\%, 41.6\%), Anuradha D et al ${ }^{11}(56.5 \%, 43.5 \%)$ and Kumar $\mathrm{R}$ et al $(59.5 \%, 40.4 \%)$. Significantly higher males were admitted in both inborn and out born unit. Gender bias as a cause of more admission to be further evaluated. A study from Uttar Pradesh found that expenditure on male is four fold higher then female ${ }^{14}$ though such study regarding gender bias is lacking in this region. In present study equal number of normal weight $(50.2 \%)$ and low birth weight $(49.7 \%)$ neonates were admitted

Table 7: Proportionate mortality

\begin{tabular}{|c|c|c|c|c|}
\hline Category & Inborn & Out born & Total & Parameters \\
\hline \multirow{4}{*}{$\begin{array}{l}\text { Birth } \\
\text { weight }\end{array}$} & $>2500 \mathrm{gm}$ & $149 / 1861(8.0 \%)$ & $119 / 976(12.19 \%)$ & $268 / 2837(9.4 \%)$ \\
\hline & $1500-2499 \mathrm{gm}(\mathrm{LBW})$ & $139 / 1507(9.2 \%)$ & $90 / 721(12.48 \%)$ & $229 / 2228(10.2 \%)$ \\
\hline & 1000-1499 (VLBW) & $58 / 259(22.3 \%)$ & $57 / 260 \quad(21.9 \%)$ & $115 / 519 \quad(22.1 \%)$ \\
\hline & $<1000 \mathrm{gm}(\mathrm{ELBW})$ & $17 / 30(56.6 \%)$ & $20 / 35(57.1 \%)$ & $37 / 65 \quad(56.9 \%)$ \\
\hline
\end{tabular}

LBW = Low birth weight, VLBW = Very low birth weight, ELBW = Extremely low birth weight

72 hours of admission (table 5). Birth asphyxia was the major cause of mortality which was $53.9 \%$ (inborn $57.8 \%$ and out born $48.9 \%$ ). It was followed by Pre term RDS in $23.2 \%$ (inborn $22.3 \%$ and out born $24.4 \%$ ) and Sepsis in $12.4 \%$ (inborn $7.7 \%$, out born $18.5 \%$ ). Other causes of death were MAS $3.2 \%$, Miscall nous (other) causes $6.7 \%$. Congenital malformation as a cause of mortality was only $0.15 \%$ (table 6). Proportionate mortality according to the birth weight was that with decreasing birth weight mortality increases significantly. In inborn unit, mortality for normal weight baby was only $8 \%$ which increases with decreasing weight like in LBW $9.2 \%$, VLBW $22.3 \%$ and ELBW $56.6 \%$. Among out born patient mortality in normal weight was $12.19 \%$ which also increases with decreasing weight as in LBW $12.48 \%$, VLBW $21.9 \%$ and ELBW $56.6 \%$ of cases (table 7).

\section{Discussion}

In our study $64.7 \%$ of the babies were inborn while $35.2 \%$ were out born which is comparable to other studies like Kumar R et $\mathrm{al}^{9}$ (inborn $60.8 \%$, out born 39.2\%), Saharia and the rest were preterm. Among preterm, babies between 34-37 weeks gestation was $33.1 \%$ whereas $17.4 \%$ baby was $<34$ weeks of gestation. This finding is comparable to Rakholia et $\mathrm{al}^{13}$ (Term 49.6\%, 34-37 weeks-42.4\% and $<34$ wks $7.9 \%$ ) but findings were totally opposite to Kumar R et al (term $64.7 \%$, pre term $35.2 \%$ ). The common morbidities seen in the admitted neonates were Birth Asphyxia (28.7\%), Jaundice requiring phototherapy (19.9\%), other (22.4\%) and Sepsis $(10.8 \%$. Other neonates include all LBW neonates below $1800 \mathrm{gm}$ which require special care. The common causes of morbidly were similar across various studies $5,7,9,13$. Higher incidence of birth asphyxia is due to late arrival of the patient for delivery leading to unplanned intervention or delayed intervention, delayed referral from periphery, delayed treatment because of high delivery load with limited human resources. In our study , high incidence of sepsis is seen in out born ( $12.9 \%)$ than inborn ( 9.6\%) patients which is comparable as in Saharia NP et al (out born 18.4\% and inborn $8.6 \%$ ) and Ranjan $\mathrm{A}$ et al ${ }^{10}$ (out born $17.2 \%$, inborn $8.5 \%$ ). The high incidence of sepsis in out born is 
related to unhygienic delivery practices in the periphery, overcrowding of neonatal unit, lack of adequate manpower and non compliance with asepsis protocol during neonatal care. The incidence of neonatal jaundice in present study is $19.9 \%$ (inborn $20.5 \%$ and out born $18.6 \%$ ) which is different across the studies as shown by Saharia NP et al $26.6 \%$, Kotwal YS et al ${ }^{11} 26.7 \%$, Malik S et al $14.9 \%$, Rakholia R et al $12.9 \%$. Other causes for admission were pre term RDS (5.25\%), MAS(4.6\%), other cases of respiratory distress like TTNB (2.26\%), hypothermia (1.4\%), hypoglycemia (3.7\%) and congenital malformation $(0.69 \%)$. Since there was no facility of pediatric surgery for surgical intervention of neonates, only few cases $(0.69 \%)$ of congenital malformation were admitted. Out of the total 5649 admitted neonates, 161 (2.8\%) patients were referred to other centre while 461(8.1\%) patients left against medical advice (LAMA). Out of the remaining 5027 babies, 4378(77.5\%) were discharged successfully and 649(11.4\%) neonates expired. Rate of successful discharge is less than Baruah MN et al (83.3\%) but more than Rakholia R et al $66.5 \%$ and NNPD ${ }^{3} 69.3 \%$. Incidence of LAMA is comparable to other studies like Rakholia et al 8.3\%, Baruah MN et al (7.5\%) and NNPD (7.5\%). The mortality rate in the present study is $11.4 \%$ (inborn $9.9 \%$ and out born $14.2 \%$ ). Mortality rate varies among different studies which depend on infrastructure of the treating facility and also the condition of the patient at the time of admission. Out born mortality is much higher than inborn mortality. This reflects the issues like delayed referral, lack of pre transport stabilization, in adequate functioning of peripheral neonatal stabilization units ((NBSU.) The Mortality rate of our study is higher than other study as shown by Baruah et al $7.5 \%$, Kumar R et al $8.1 \%$, Kotwal YS et al $9.7 \%$, but lower than NNPD $16.9 \%$, Rakholia R et al $20.53 \%$ and Ranjan A et al $23.4 \%$.

Gender specific mortality is that out of 649 death, $58.7 \%$ (inborn $58.0 \%$ and out born $58.7 \%$ ) are male and $41.2 \%$ (inborn $41.9 \%$ and out born $41.2 \% \%$ ) are female. Among the total mortality of $11.4 \%$, male contribution is $6.7 \%$ and female is $4.7 \%$. Term babies were more than Preterm (56.7\% vs $43.2 \%$ ) which similar to studies done by Kumar $\mathrm{R}$ et al $(59.6 \%$ vs $40.3 \%)$. Analysis of age at birth shows that it occurred $88.5 \%$ in early neonates and only $11.4 \%$ in late neonatal period which is similar to Baruah $\mathrm{MN}$ et al which found that $77.5 \%$ death occurred in early neonatal period. . Birth weight specific mortality shows that it is more in low birth weight babies $(58.9 \%)$ in comparison to normal weight (41.2\%). Trend is similar in both out born and inborn babies.
The present study is similar to other studies like Ranjan et al where normal weight baby among dead was $46.2 \%$ and LBW was $53.7 \%$ but Baruah MN et al published much higher prevalence of mortality among low birth weight babies which is $70.6 \%$. Analysis of time interval between admission and death revealed that it took place in $27.4 \%$ of cases within 24 hours of admission, in between 1-3 days $50.8 \%$ and in $4-7$ days $15.5 \%$. Beyond 7 days of admission it was only $6.1 \%$. .Thus $78.2 \%$ of death occurred within 72 hours of admission. Similar trend was shown by Kumar et al which showed that $68.9 \%$ death was within 72 hours of admission.

Disease specific mortality revealed that three common causes of death are birth asphyxia (53.9\%), RDS with prematurity $(23.2 \%$ and sepsis $(12.4 \%)$ which is similar to different studies $(5,7,9,10,13)$ though incidence of these three causes vary among studies. Incidence of mortality due to birth asphyxia in our study was $53.9 \%$ (inborn $57.8 \%$ and out born 48.9\%) which much higher than Baruah MN et al (29.3\%) Ranjan et al ${ }^{10}(20.84 \%$, Kumar R et al $36.4 \%$ but similar to some other study like Saharia NP et al (57\%), a study done at Guwahati Medical college, the region's premier teaching institute. This high incidence of birth asphyxia may be due to late arrival of patients to the facility for seeking treatment leading to unplanned interventions, referral of all complicated cases to the hospital due to lack of other such well equipped and cheap facility in the area, delay in taking interventions during delivery and resuscitation due to high patient load and low care giver.

Incidence of death due to RDS with prematurity is 23.2 \% (inborn22.3\% outborn24.4\%) which is similar to Saharia NP et al (19.8\%), Kumar R et al (17.05\%) and Baruah MN et al $15.9 \%$. Overall incidence of Sepsis is $14.9 \%$ which is similar to Kotwal YS et al (18.2\%), Saharia et al $15.7 \%$, Ranjan A et al $17.9 \%$. Present study also shows much higher incidence of mortality due to sepsis in out born (18.5\%) compared to inborn (7.7\%). This trend of high out born sepsis is published by some other studies also like Kumar $\mathrm{R}$ et al (out born 15.2\%, inborn $8.6 \%$,). High incidence of sepsis is due to unhygienic delivery practices in the periphery, overcrowding of neonatal unit, lack of adequate manpower and non compliance with asepsis protocol during neonatal care. Other causes of mortality were MAS 3.2\%, miscellaneous (other) causes 6.7\%. Congenital malformation as a cause of mortality is only $0.15 \%$. It is because of non availability of the pediatric surgery unit, only few cases of congenital anomalies were admitted, others were referred to 
other facility. Proportionate mortality reveals that lower the birth weight higher the probability of death. Mortality of baby with birth weight 2500 gm or more is only $9.4 \%$ which increases to $10.2 \%$ in the babies whose weight is $1500-$ $2499 \mathrm{gm}$. But in the babies with 1000 - 1499gm it becomes $22.1 \%$ and below 1000 gm babies death is almost half of admitted babies $(56.9 \%)$. Similar trend is also shown by Baruah MN et al and Modi $\mathrm{R}$ et $\mathrm{al}^{6}$ also.

Limitation of the study: The present study is a hospital based retrospective study. Therefore like prospective study we could not analyze the epidemiological factors related to neonatal health especially socio economic back ground, maternal antenatal, intra natal and post natal factors that could have influenced the outcome.

\section{Conclusion}

Our study shows that birth asphyxia, jaundice and sepsis are common morbidities requiring admission. Birth asphyxia is the commonest cause of mortality followed by RDS with prematurity and neonatal sepsis.

\section{Conflict of interest: None. Disclaimer: Nil.}

\section{References}

1. UNICEF, WHO, World Bank Group and United Nations. Levels and trends in Child Mortality 2019. Zeneva: WHO; 2019.

2. Ministry of Health and Family Welfare. National Family Health Survey-4 reports. Mumbai: International Institute for Population Sciences; 2017.

3. NNPD Network, Indian Council of Medical Research, National Neonatology Forum (India). National Neonatal Prenatal Database: Report 2002-2003. New Delhi: Nodal Centre, AIIMS; 2005.

4. Nonita D, Sankalp D, Jyoti K, Damodar B. Chilhood mortality and mortality in India- Analysis of National Family Health Survey 4 (NFHS-4) findings. Indian Pediatrics. 2018; 55(4): 335-8.

5. Saharia NP, Deka A, Vivekananda MS. Mortality and Morbidity at tern of Neonatal ICU of Guwahati Medical College and Hospital: IOSR Journal of Dental and Medical Sciences. 2016;15(6): 73-5.

6. Rohit M, Bhabesh M, Jaimin Kumar P, Margareth Punitha K. Study of the Morbidity and mortality Pattern in the Neonal Intensive Care unit at a tertiary care teaching Hosital in Gandhinagar District, Gujarat, India. Journal of Research in Medical and Dental Science. 2015; 13(3):208-12.
7. Baruah MN, Panyang PP. Morbidity and mortality Profile of Newborns Admitted to the Special Care Newborn Unit (SCNU) of a Teaching Hospital of Upper Assam, India- A Three Year Study. Journal of Medical Science and Clinical Research. 2016; 4(8): 11689-95.

8. Malik S, Gohia P, Khan IA. Morbidity Profile and Mortality of Neonates Admitted in Neonatal Intensive Care Unit of a Centraal India Teaching Institute: A Prospective Observational Study. Journal of Clinical Neonatology. 2016; 5(3): 168-73.

9. Kumar R, Mundhra R, Jain A, Jain S. Morbidity and mortality profile of neonates admitted in special newborn care unit of a Teaching Hospital in Uttarakhand, India. Internatinal Journal of Research in Medical Sciences. 2019; 17(1): 241-6.

10. Ranjan A, Singh A. Pattern of morbidity and mortality of neonates admitted in tertiary level neonatal intensive care unit in Nalanda Medical College and Hospital, Bihar, India. Internatinal Journal of Contemorary Pediatrics. 2016; 13: 854-7.

11. Anuradha D, Rajesh Kumar S, Aravind MA, Jayakumar M, Ganesh J. A profile on the spectrum of neonatal mortality and morbidity at tern of extramural neonates in the Specialised Neonatal Care Unit (SCNU) in a tertiary care hospital. International Journal of Contemporary Pediatrics. 2018; 15(2): 427-31.

12. Kotwal YS, Yatoo GH, Jan FA. Morbidity and Mortality Among Neonates Admitted to a Neonatal Intensive care Unit of a Tertiary Care Teaching Hospital of Jammu and Kashmir, India. Neonat Pediatr Med. 2017; Vol 3(2): 136

13. Rakholia R, Rawat V, Bano M, Singh G. Neonatal morbidity and mortality of sick newborns admitted in a teaching hospital. CHRISMED Journal of Health and Research. 2014; 11(4): 228-34.

14. Willis JR, Kumar V, Mohanty S, Singh P, Singh V, Baqui AH. Gender differences in perception and care seeking for illness of newborns in rural Uttar Pradesh, India. J Health Population Nutr. 2009; 27 (1): 62-71.

\footnotetext{
Kutubur Rahman ${ }^{1}$, Rukeya Begum ${ }^{2}$

${ }^{1}$ Assistant Professor; ${ }^{2}$ Associate Professor , Department of Pediatrics, Tezpur Medical College , Tezpur, Assam, India.
} 